\title{
Editorial
}

\section{Two-Dimensional Materials Based Optoelectronics}

\author{
Yu Chen, ${ }^{1}$ Jun Liu, ${ }^{2}$ Jizhou Jiang, ${ }^{3}$ Xiaofeng Zhou, ${ }^{4}$ Shuqing Chen, ${ }^{1}$ and Yan Luo ${ }^{5}$ \\ ${ }^{1}$ International Collaborative Laboratory of 2D Materials for Optoelectronics Science and Technology, Key Laboratory of \\ Optoelectronic Devices and Systems of Ministry of Education and Guangdong Province, College of Optoelectronic Engineering, \\ Shenzhen University, Shenzhen 518060, China \\ ${ }^{2}$ Aston Institute of Photonic Technologies, School of Engineering and Applied Science, Aston University, Birmingham B4 7ET, UK \\ ${ }^{3}$ Department of Physics, National University of Singapore, 2 Science Drive 3, Singapore 117542 \\ ${ }^{4}$ Physical Sciences and Engineering Division, King Abdullah University of Science and Technology, Thuwal 23955-6900, Saudi Arabia \\ ${ }^{5}$ Department of Chemical Engineering, West Virginia University, 313 Engineering Research Building, Evansdale Drive, \\ Morgantown, WV 26506, USA \\ Correspondence should be addressed to Yu Chen; yuchen@szu.edu.cn
}

Received 24 September 2017; Accepted 25 September 2017; Published 28 November 2017

Copyright (C) 2017 Yu Chen et al. This is an open access article distributed under the Creative Commons Attribution License, which permits unrestricted use, distribution, and reproduction in any medium, provided the original work is properly cited.

Recently, originating from the emerging and intensive research of graphene, there have been tremendous improvements in the area of two-dimensional (2D) materials (e.g., graphene, topological insulators, and black phosphorus) based optoelectronics. Their unique and novel characteristics strongly promoted the developments of condensed-matter physics, device physics, photonics, electronics, optoelectronics, and so on. The purpose of this feature issue is to present the state of recent progresses in the field, from fundamental physics (condensed-matter physics, device physics, and so on) to various applications of the emerging $2 \mathrm{D}$ materials. The feature issue covers various aspects of theoretical and experimental researches related to $2 \mathrm{D}$ materials based optoelectronics. Among plenty of submissions, we selected 8 papers for publication in the special issue.

The paper posted by S. Chen et al. demonstrates a homemade nonlinear response measurement system of 2D materials in sub-terahertz waveband. Using mutual frequency beating technology, they achieve the radiation of $0.1 \mathrm{THz}$ wave with power up to $4 \mathrm{~mW}$. Based on this sub-terahertz source, they measure the nonlinear response of topological insulator. The paper by G. Li et al. studies the optical group delay in a modified Otto configuration with graphene surface plasmon. The optical group delay can be tuned obviously, even from negative to positive, with variety of means (Fermi energy, relaxation time of graphene, distance between the coupling prism, and graphene). The paper by Z. Zeng et al. investigates the bistability in one-dimensional photonic crystal (1DPC) covered by graphene. By tuning the Femi energy and the number of graphene monolayers, the hysteresis and nonlinear lateral shift in 1DPCs can be controlled effectively.

The paper by Y. Cai proposes a submicrosecond passively Q-switching Er-doped fiber laser with black phosphorus (BP) as saturable absorber (SA). By taking full advantage of the ultra-fast relaxation time of BP SA and careful design of compact ring cavity, they obtain stable Q-switching pulses output with record duration of $742 \mathrm{~ns}$ experimentally. It indicates that BP-SA can be an effective SA for nanosecond Qswitching pulse generation. The paper by J. Wu et al. reports a high-power cladding-pumped Er,Yb codoped all-fiber laser with truly single transverse mode output. By using a pair of fiber Bragg gratings (FBGs), two pieces of standard singlemode fibers, and other careful cavity designs, they obtain a record high of output power with single-mode in an all-fiber single-mode Er,Yb codoped laser. The work by Z. Deng et al. investigates the generation of dispersive wave (DW) in normal group-velocity dispersion (GVD) region with photonic crystal fiber (PCF). Through controlling DW generation in gas-filled PCF with combined effects of self-steepening (SS) and chirp, one can achieve any wavelength artificially with controllable energy.

The paper by $\mathrm{M}$. Su et al. proposes a novel equalizing Dammann vortex grating (EDVG) to realize orbital angular momentum (OAM) signal demultiplexing and channel 
equalization. By suppressing odd diffraction orders and adjusting grating structure, the total diffraction efficiency of EDVG can be highly improved. Therefore, the bit error rate (BER) of each OAM channel can be decreased sharply.

The paper by Y. Chen et al. demonstrates random optical field with special autocorrelation which is different from the general autocorrelation with a Gaussian function. The speckle with special autocorrelation has self-healing effect. The selfhealing process is affected by the size of obstruction and the coherence of the speckle but is almost not affected by the shape of obstruction.

Yu Chen

Jun Liu Jizhou Jiang

Xiaofeng Zhou

Shuqing Chen

Yan Luo 

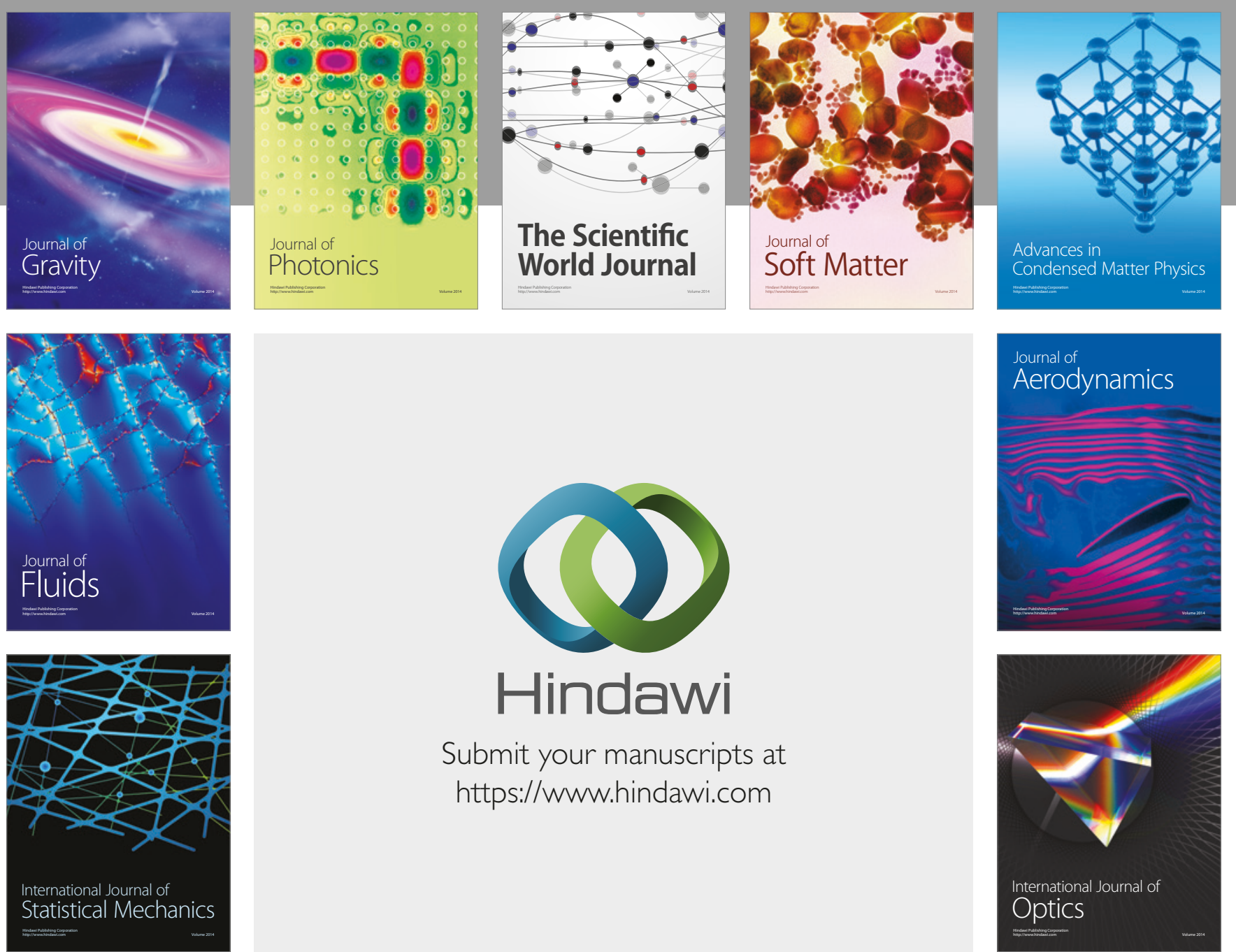

Submit your manuscripts at

https://www.hindawi.com
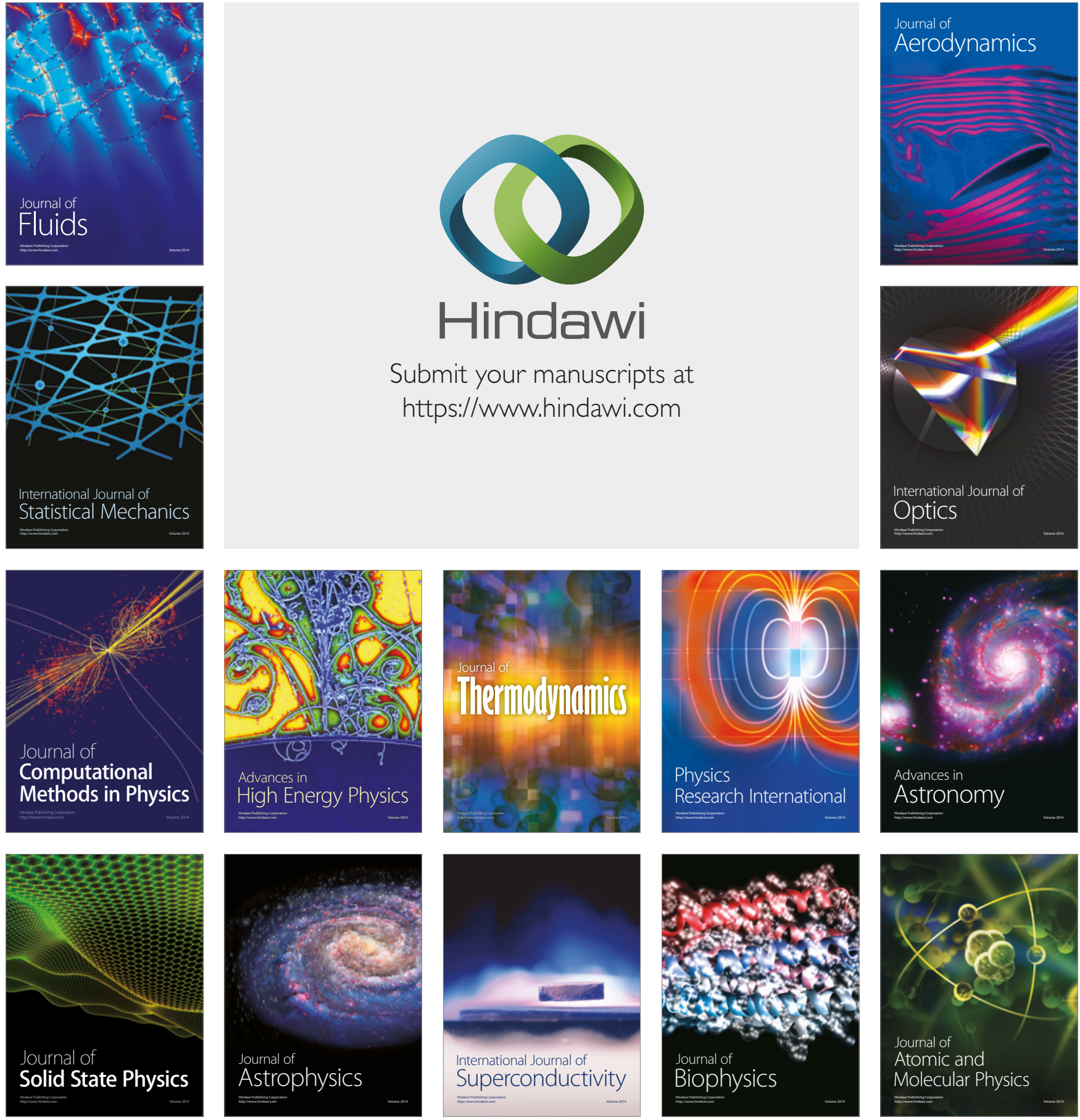